\title{
Foreword to the
}

\section{Proceedings of the CEMRACS 2013}

This volume contains contributions related to the CEMRACS 2013 devoted to: Modelling and simulation of complex systems: stochastic and deterministic approaches. The web page of the conference can be found at the address

http://smai .emath.fr/cemracs/cemracs13/

The CEMRACS (Centre d'Eté Mathématique de Recherche Avancée en Calcul Scientifique) is an annual summer research session for $\mathrm{PhD}$ students and young researchers, whose goal is to strengthen interdisciplinary collaborations between applied mathematicians and scientists of other fields, both from academia and industry. It is promoted by the SMAI (French Society of Applied and Industrial Mathematics) and was initiated by Frédéric Coquel and Yvon Maday in 1996.

In 2013, the CEMRACS 2013 was organized by Nicolas Champagnat (INRIA Nancy - Grand Est), Tony Lelièvre (Ecole des Ponts ParisTech) and Anthony Nouy (Ecole Centrale de Nantes). It took place at the CIRM (Centre International de Rencontres Mathématiques, Marseille, France) from July 22th to August 30th, 2013. The goal was to bring together scientists from both the academic and industrial communities and discuss topics related to the modelling and the simulation of complex systems, using both stochastic and deterministic approaches. The main application themes were: uncertainty propagation, molecular and multiscale simulation, and population dynamics. The mathematical models and numerical methods relevant to this conference were: advanced Monte Carlo methods, stochastic partial differential equations and numerical methods for high dimensional problems.

The CEMRACS 2013 consisted in two joint events: a summer school (July 22 - July 26) and then an intensive five weeks long research session (July 29 - August 30). During the summer school, five lectures were given by: Albert Cohen (High dimensional stochastic-parametric PDE's: Theory and Algorithm), Josselin Garnier (Rare events: models and simulations), Gabriel Lord (Stochastic Partial Differential Equations), Felix 
Otto (Stochastic Homogenization and Random Materials) and Gilles Pagès (Quantification and Quasi Monte Carlo methods). In addition, a few contributed talks were devoted to some specific subjects, given by Sylvain Arlot, Didier Auroux, Freddy Bouchet, Boris Khoromskij, Régis Lebrun and Gianluigi Rozza. Finally, an afternoon session dedicated to the special year "Mathematics of Planet Earth" and co-organized with the ARP MathsInTerre was organized with two lectures by Alexandre Ern and Sylvie Méléard, and a round table animated by Didier Bresch with four invited speakers: Denis Allard (INRA), Nadai Maïzi (Mines ParisTech), Frédéric Plas (ANDRA) and Verena Trenkel (IFREMER). We would like to acknowledge funding support from CNRS, Fondation IXCore and INRIA for the organization of the summer school.

During the research session, 16 research projects were investigated by 37 young researchers. The research session was supported by private funding (CEA, EDF, IFPEN, IRSN, ONERA, Sanofi, Safety Line) and public funding (Labex AMIES, Labex Bezout, CNRS grand défi NEEDS, Labex MMCD, Polytech'Nice). We warmly thank our sponsors for this event.

This issue of ESAIM: Proceedings and Surveys consists of 4 contributions related to the summer school and 16 articles resulting from the research projects initiated during the research session. All the authors of these 20 articles are thanked for their contributions. For this volume, we proposed to each reviewer to publish a (signed or anonymous) summary of the report, in order to provide some introductory materials to the articles. We hope that the readers will find these supplementary texts useful.

In addition to the numerous sponsors mentioned above, we would like to thank the HPC resources of Aix-Marseille Université financed by the project Equip@Meso (ANR-10-EQPX-29-01) which were used by some of the projects, and the people who helped us for the organization of this event: Pauline Lafitte, Frédéric Lagoutière, Pierre Navaro, Alain Prignet, Noura Sahtout, and the CIRM staff. Finally, we would like to address our warmest thanks to all the participants, who contributed to making this event a success.

The organizing committee: Nicolas Champagnat, Tony Lelièvre and Anthony Nouy. 